\title{
Intelligent Torque Vectoring Approach for Electric Vehicles with Per-Wheel Motors
}

\author{
Alberto Parra $\mathbb{D}^{\mathbb{D}},{ }^{1}$ Asier Zubizarreta $\mathbb{D}^{\mathbb{D}},{ }^{2}$ Joshué Pérez, ${ }^{1}$ and Martín Dendaluce ${ }^{2}$ \\ ${ }^{1}$ Tecnalia Research \& Innovation, Industry and Transport Division, Donostia, Spain \\ ${ }^{2}$ Department of Automatics and System Engineering, Faculty of Engineering of Bilbao, University of the Basque Country (UPV/EHU), \\ Bilbao, Spain \\ Correspondence should be addressed to Alberto Parra; alberto.parra@tecnalia.com
}

Received 24 November 2017; Revised 15 January 2018; Accepted 23 January 2018; Published 25 February 2018

Academic Editor: José Manuel Andújar

Copyright (C) 2018 Alberto Parra et al. This is an open access article distributed under the Creative Commons Attribution License, which permits unrestricted use, distribution, and reproduction in any medium, provided the original work is properly cited.

\begin{abstract}
Transport electrification is currently a priority for authorities, manufacturers, and research centers around the world. The development of electric vehicles and the improvement of their functionalities are key elements in this strategy. As a result, there is a need for further research in emission reduction, efficiency improvement, or dynamic handling approaches. In order to achieve these objectives, the development of suitable Advanced Driver-Assistance Systems (ADAS) is required. Although traditional control techniques have been widely used for ADAS implementation, the complexity of electric multimotor powertrains makes intelligent control approaches appropriate for these cases. In this work, a novel intelligent Torque Vectoring (TV) system, composed of a neuro-fuzzy vertical tire forces estimator and a fuzzy yaw moment controller, is proposed, which allows enhancing the dynamic behaviour of electric multimotor vehicles. The proposed approach is compared with traditional strategies using the high fidelity vehicle dynamics simulator Dynacar. Results show that the proposed intelligent Torque Vectoring system is able to increase the efficiency of the vehicle by $10 \%$, thanks to the optimal torque distribution and the use of a neuro-fuzzy vertical tire forces estimator which provides 3 times more accurate estimations than analytical approaches.
\end{abstract}

\section{Introduction}

The need for reducing global warming, air pollution, and oil dependency has motivated not only the use of renewable energies, but also some paradigm changes in other areas, such as transportation systems, where the development of electric vehicles (EV) has become a key strategy [1]. The interest in vehicles with electrified powertrains (fully electric as well as hybrid) has increased in the last years, becoming one of the main research areas in the automotive industry [2].

The integration of electric motors in propulsion systems provides not only better energy efficiency and lower pollution, but also increased controllability, as electric motors offer better response time [3]. These features are fueling a notable interest in the development of Advanced DriverAssistance Systems (ADAS) that enhance not only the dynamic behaviour of the vehicle, but also its efficiency and energy consumption [4].
Traditional control approaches have been widely used to implement ADAS during the last decades. However, electrified propulsion systems offer wider complexity (and multiple topologies) than internal combustion propulsion systems. Due to this, intelligent control approaches have become one of the main research interests lately, as they can manage complex systems more easily than traditional approaches.

One of the most complete ADAS for enhancing the dynamic behaviour and stability of an electric vehicle with per-wheel motors is Torque Vectoring (TV) [5], which focuses on the optimal driving torque distribution. Several strategies can be used to control the torque distribution in a TV approach, with most of them being based on controlling the moment along the vertical axis of the vehicle (yaw moment) [6]. For this purpose, three main strategies are used [7]: the first one is based on the distribution of the torque among the driving wheels $[6,8-12]$; the second one is based 
on using active vehicle roll control systems to vary the lateral load distribution $[13,14]$; finally, the third strategy consists in implementing a steering system in both axles $[15,16]$. This work focuses on torque distribution strategies, as an all-wheel drive vehicle has been selected as case study.

Torque distribution approaches have been implemented conventionally using a wide variety of control algorithms. Among the traditional approaches, simpler ones, such as proportional-integral-derivative control (PID) based ones [6], or more advanced algorithms, such as Model Predictive Control (MPC) [17] or Sliding Mode Control (SMC) [9], have been proposed. The latter provide enhanced results, although their computational cost is higher than PID based approaches. On the other hand, intelligent approaches, such as neural networks [10] or fuzzy logic systems [11], have been demonstrated to be able to provide good results with lower computational cost, making them a good alternative to the implementation of torque distribution approaches.

In order to achieve an effective driving torque distribution, the knowledge of the tire forces is crucial [18]. Direct measurement of these forces, however, is a complex and difficult task, and, therefore, the design of proper estimators is required. Nevertheless, this issue is not always considered in the works proposed in the literature, which are based on perfect estimations of these forces, which is not a real-case scenario.

Among the approaches proposed to estimate the vehicle tire forces, the most common one is the use of estimators based on tire models, such as the linear tire model [19], Dugoff's model [20], or semiempirical models such as the Pacejka's tire model [18]. Other works do not consider the tire model and estimate vertical tire forces based on longitudinal and lateral load transfers as well as the static loads on each wheel [21-25]. Intelligent modelling approaches have also been used, which reduce the need for knowing the complex tire dynamics and even allow model adaptation. For instance, neural networks, extended Kalman filters, and recursive least squares approaches are combined in [26] to estimate tire lateral force and grip potential identification even in aggressive manoeuvres. In [27] a longitudinal, lateral, and vertical tire force estimator based on fuzzy logic is used, which requires the slip angle estimation to operate and provides high correlation. Both approaches provide more accurate estimations compared with traditional approaches, although their applicability presents some inconveniences, as they require variables difficult to measure.

In summary, intelligent approaches have been demonstrated to be a suitable alternative to ADAS development, providing balanced performance versus computational cost. However, proper tire force estimations are required to guarantee this theoretical performance in a real-case scenario. In the literature, most works consider perfect estimations or use estimators based on physical variables difficult to measure, which require expensive sensors, or use complex models whose parameters are difficult to identify. This issue reduces the implementability and performance of the approaches proposed in most works in real-case scenarios.

In order to solve these issues, this work presents a novel intelligent Torque Vectoring approach, composed of two intelligent algorithms: first, an adaptive neuro-fuzzy inference system (ANFIS) estimator for the tire vertical forces based on exclusively measurable variables; second, a fuzzy yaw moment controller, which controls both vehicle yaw rate and sideslip angle, as they are some of the most representative vehicle dynamics variables. The proposed approach is able to enhance electric vehicle dynamics and their efficiency. To demonstrate its effectiveness, the ANFIS estimator and the resulting intelligent TV system have been validated considering several scenarios in the Dynacar high fidelity dynamic simulator, using an E-Class vehicle and comparing the obtained results with other previous works from the literature.

The rest of the paper is divided as follows: In Section 2 the proposed intelligent Torque Vectoring approach is detailed. In Section 3, the simulation framework and experimental setup are presented. In Section 4, the results of the validation carried out are explained. Finally, in Section 5, the main conclusions are presented.

\section{Intelligent Torque Vectoring System}

In this section the proposed intelligent Torque Vectoring system is detailed. Its main purpose is to distribute the driving torque among the different actuated wheels, so that vehicle handling and stability is improved. It can be divided into 5 subsystems (Figure 1): the lateral torque distribution is carried out using a fuzzy yaw moment controller, whose reference is calculated using a yaw rate reference generator; the longitudinal torque distribution is given by a self-defined torque distribution algorithm, which uses an ANFIS vertical tire forces estimator; the data provided by the longitudinal and lateral distributions is used to calculate the final torque distribution.

2.1. Yaw Rate Reference Generator. The developed intelligent TV system approach is composed of a lateral torque distribution approach and a longitudinal one. The first is based on the control of the yaw moment of the vehicle; this is, it requires an appropriate yaw rate reference for its proper performance.

For the calculation of the desired yaw rate reference, the well-known bicycle model is used, as it provides a good balance between accuracy and computational cost [28]. In order to further increase computational performance, the following assumptions and simplifications are carried out: the center of gravity is assumed to be at a height of zero; the variation of the vertical force of each tire will not be taken into account; small slip angles assumption will be considered, so that $\sin (\beta)=\beta$ and $\cos (\beta)=1$ (linear region); and the coefficient of lateral stiffness of the tire will be constant, defined by the ratio of the lateral force to the slip angle.

It must be noted that this model of reduced complexity is exclusively used for real-time execution in the controller. In addition, some of these simplifications are reasonable for passenger cars, as they are not driven until the limits of the tires. 


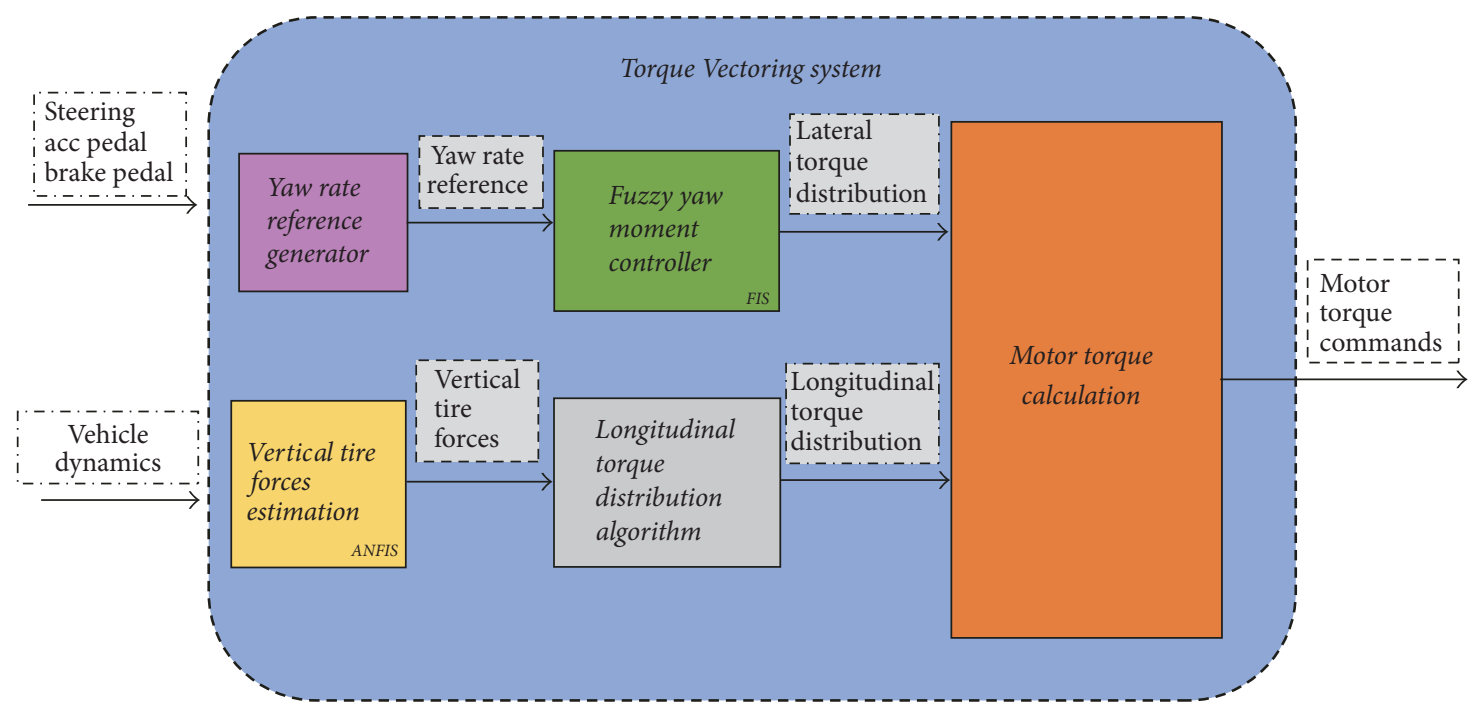

FIGURE 1: Proposed intelligent torque vectoring system.

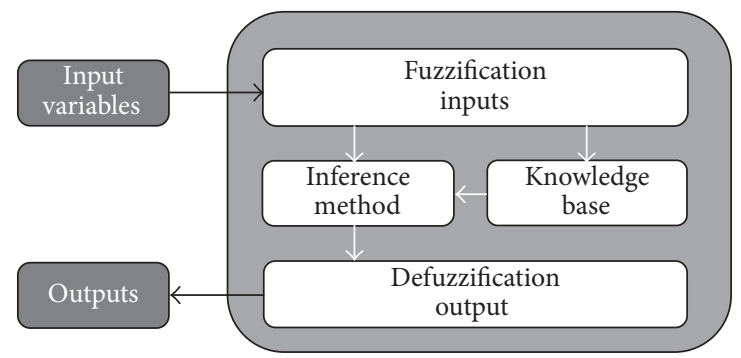

FIgURE 2: General diagram representing fuzzy logic approaches.

This way, the yaw rate reference equation is [28]

$$
\dot{\psi}_{\text {ref }}=\frac{V}{L+(m / L)\left(b / C_{\alpha F}-a / C_{\alpha R}\right) V^{2}} \delta,
$$

where $m$ is the total mass of the vehicle located in the center of gravity, $a$ and $b$ are the distance to the center of gravity of the front and rear axle, respectively, $L$ is the distance between axles, $\delta$ is the angle of rotation of the front wheels, $C_{\alpha F}$ and $C_{\alpha R}$ are the lateral stiffness coefficients of the front and rear wheels, respectively, and $V$ is the vehicle speed.

However, for safety reasons it is necessary to limit the value of the yaw rate reference generated. In this case, the limit has been set as follows [6]:

$$
\left|\dot{\psi}_{\text {ref,max }}\right|=\left|\frac{a_{y}}{V}\right|
$$

where $a_{y}$ is the vehicle lateral acceleration.

2.2. Fuzzy Yaw Moment Controller. The fuzzy yaw moment controller handles the lateral torque distribution $\left(\tau_{\text {lat }}\right)$ for the vehicle. Hence, considering both the yaw rate and slip angle of the vehicle, this system calculates the torque percentage to be applied to each side of the vehicle. This way, if $\tau_{\text {lat }}=0$, all the torque will be applied to the wheels of the right side; and if $\tau_{\text {lat }}=1$, all the torque is applied to the wheels of the left side.

This subsystem is based on fuzzy logic, which is an extension of Boolean logic by Zadeh in 1965 [29] based on the mathematical theory of fuzzy sets. It enables an abstraction from the model and mathematical formulations by translating expert knowledge into rules without renouncing a notable level of fine-tuning capabilities.

The most common fuzzy logic system structure is shown in Figure 2. First a fuzzification process must be carried out to transform the input data into fuzzy sets to continue with an inference system, based on the developed rules. Finally, a defuzzification method is necessary in order to convert the result given by the inference system into the exact value to, in this particular case, ensure appropriated control.

The proposed fuzzy logic controller is based on the Mamdani fuzzy model, as it provides a more intuitive tuning [30]. In order to calculate the torque percentage to be applied to each side of the vehicle, $\tau_{\text {lat }}$, the controller requires three inputs: the yaw rate error, its derivative, and the side slip angle error. The yaw rate error and its derivative are calculated considering the reference detailed in the previous subsection. The slip angle error is calculated considering that the reference sideslip angle of the vehicle will be zero, in 

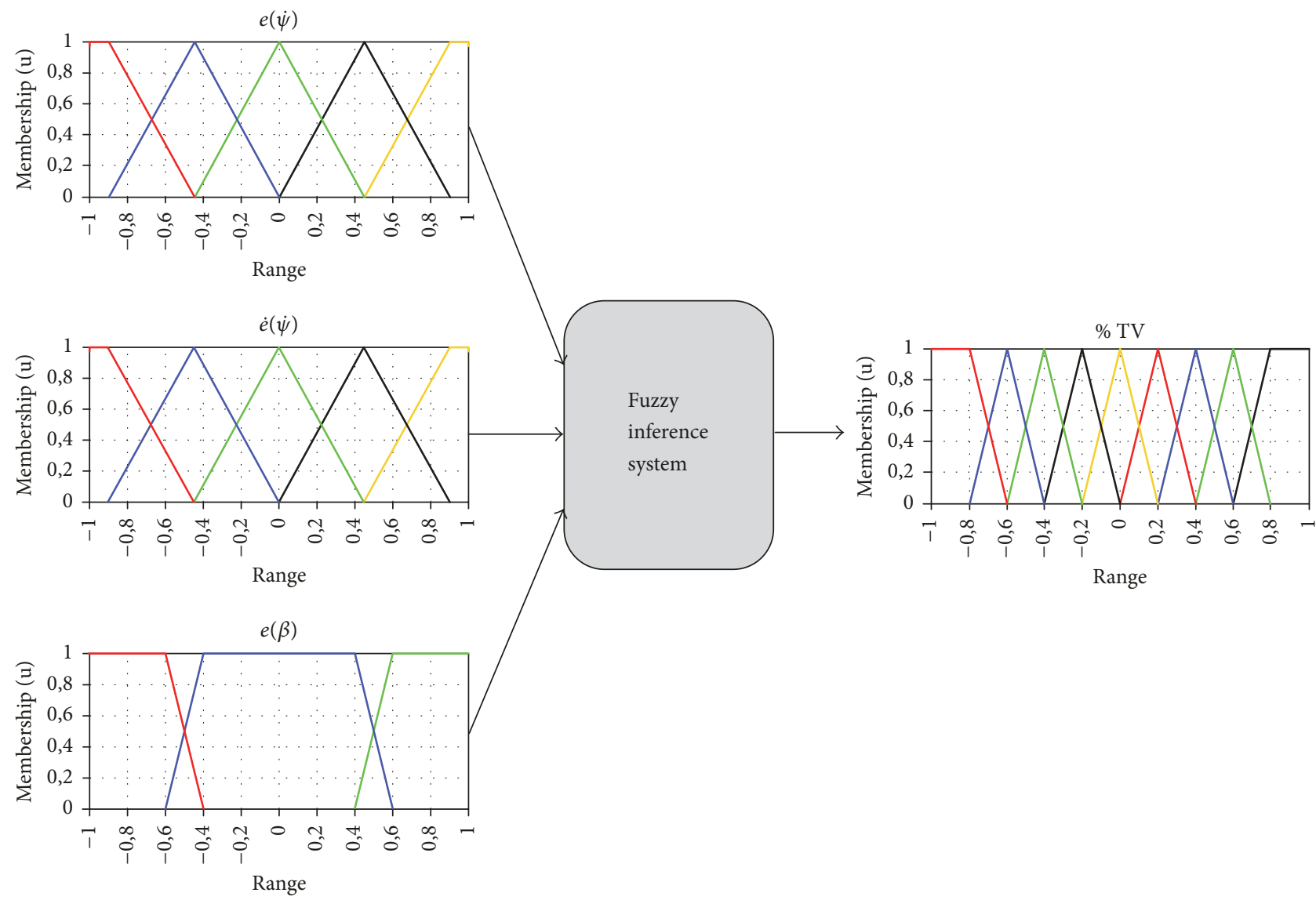

FIGURE 3: Fuzzy logic controller proposed.

order to reduce the real value of the slip and achieve a neutral handling.

The actual vehicle sideslip angle value is calculated using the following equation [31]:

$$
\beta=\arctan \left(\frac{V_{y}}{V_{x}}\right),
$$

where $V_{y}$ and $V_{x}$ are the vehicle speed in longitudinal $(x)$ and lateral $(y)$ local axes.

For the design of the fuzzy system the following structure has been implemented. First, a distribution of 5 membership functions has been chosen for the yaw rate error $e(\psi)$ and for its derivative $\dot{e}(\psi)$, and three membership functions for the input of lateral slip angle, $e(\beta)$. Considering the typical sideslip angle and yaw rate values achieved by a passenger car, it has been considered that five membership functions cover the whole range for the first two variables with a good level of accuracy. Moreover, the sign of sideslip angle determines if the vehicle has a neutral, understeering or oversteering behaviour. Therefore, it has been considered that only 3 membership functions are needed for this last variable. All of them have been selected as trapezoidal and triangular. The trapezoidal ones have been selected for the boundaries of each variable and for the membership functions of sideslip angle, since the proposed controller tries to minimize this variable and, therefore, accuracy is not the highest priority.
The triangular ones have been used for the rest of variables, as they provide computationally efficient calculations [32] maintaining acceptable smoothness on the response, suitable to be implemented in conventional automotive Electronic Control Units (ECUs).

And finally, for the output, the torque percentage to be applied to each side of the vehicle, $\tau_{\text {lat }}$, a more complex distribution of membership functions has been chosen, nine in this case, aiming to achieve a response as accurate and smooth as possible.

The structure of the developed fuzzy controller is shown in Figure 3 including the membership functions.

Subsequently the corresponding rules have been implemented based on the knowledge about the system and human driving datasets. Table 1 shows the names and description of the membership functions, while Tables 2-4 show the implemented rules.

2.3. ANFIS Vertical Tire Forces Estimator. The dynamic behaviour of the vehicle depends heavily on the tire forces, as these model the contact force between the wheels and the road. However, their estimation is one of the most complex issues in vehicle dynamics, as the tire/road contact dynamics depends on a number of different variables. Direct measurement of these forces is not always a solution either, as these forces are very difficult to measure. 
TABLE 1: Membership functions names.

\begin{tabular}{lc}
\hline Names & Description \\
\hline NVL & Negative very large \\
NL & Negative large \\
NM & Negative medium \\
NS & Negative small \\
ZE & Zero \\
PS & Positive small \\
PM & Positive medium \\
PL & Positive large \\
PVL & Positive very large \\
\hline
\end{tabular}

TABLE 2: Rules for negative yaw rate error derivative.

\begin{tabular}{ccccccc}
\hline$\dot{e}(\dot{\psi})<0$ & & \multicolumn{5}{c}{$e(\dot{\psi})$} \\
\hline \multirow{4}{*}{$e(\beta)$} & & NL & NS & ZE & $P S$ & $P L$ \\
& $N L$ & ZE & NS & NM & NL & NVL \\
& $N S$ & ZE & ZE & NS & NM & NL \\
& ZE & ZE & ZE & ZE & NS & NL \\
& $P S$ & PM & PS & ZE & ZE & NS \\
& $P L$ & PL & PM & PS & ZE & ZE \\
\hline
\end{tabular}

TABLE 3: Rules for zero yaw rate error derivative.

\begin{tabular}{ccccccc}
\hline$\dot{e}(\dot{\psi})=0$ & \multicolumn{6}{c}{$e(\dot{\psi})$} \\
\hline \multirow{4}{*}{$e(\beta)$} & & NL & NS & ZE & PS & $P L$ \\
& $N L$ & ZE & NS & NM & NL & NVL \\
& NS & PS & ZE & NS & NM & NL \\
& ZE & PM & PS & ZE & NS & NM \\
& $P S$ & PL & PM & PS & ZE & NS \\
& $P L$ & PVL & PL & PM & ZE & ZE \\
\hline
\end{tabular}

TABLE 4: Rules for positive yaw rate error derivative.

\begin{tabular}{ccccccc}
\hline$\dot{e}(\dot{\psi})>0$ & \multicolumn{6}{c}{$e(\dot{\psi})$} \\
\hline \multirow{4}{*}{$e(\beta)$} & & NL & NS & ZE & PS & $P L$ \\
& $N L$ & ZE & ZE & NS & NS & NM \\
& $N S$ & PS & ZE & ZE & NS & NS \\
& ZE & PM & PS & ZE & ZE & ZE \\
& PS & PL & PM & PS & ZE & ZE \\
& PL & PVL & PL & PM & PS & ZE \\
\hline
\end{tabular}

In this section, a novel ANFIS vertical tire forces estimator is proposed. The proposed estimator provides realtime and accurate estimations of the tire forces, which can be exploited by ADAS to increase the safety, stability, and efficiency of vehicles. Hence, this estimator will be used to perform the longitudinal dynamics torque distribution.

The proposed estimator is based on an ANFIS that is based on a fuzzy system that uses a learning algorithm derived from neural network theory to determine its parameters (fuzzy sets and fuzzy rules) by processing data samples [33]. For that purpose, the Takagi-Sugeno model is used, as it

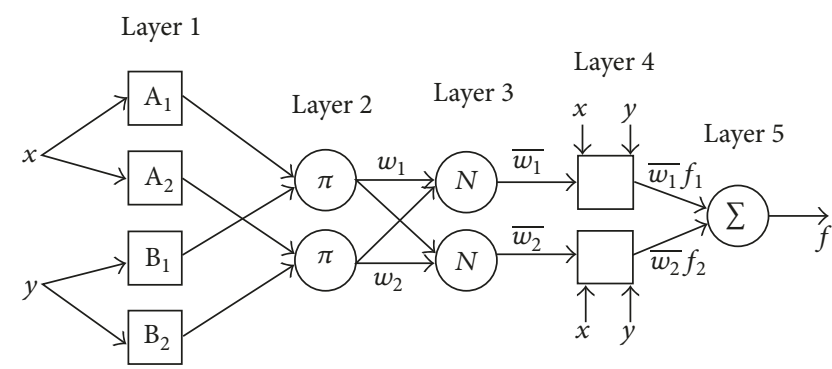

FIGURE 4: ANFIS structure [2].

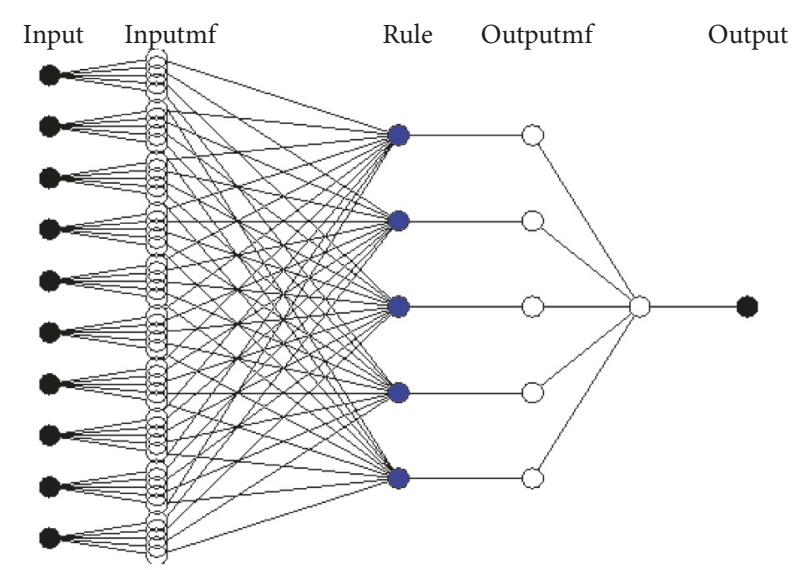

Logical operations

- And

FIGURE 5: ANFIS estimators structure.

is computationally efficient [34] and has been demonstrated to be appropriate for modelling applications. This approach maintains the fuzzy logic capability of converting human knowledge into a quantitative process and rules while it solves the membership functions iterative tuning process problem thanks to the neural networks learning ability. A simplified diagram of an ANFIS structure is shown in Figure 4.

The proposed estimator uses measurable variables to operate, which is one of the main contributions of this work compared with those analyzed in the bibliography. The input data is composed of 10 variables: the steering angle; the $x$, $y$, and $z$ linear acceleration and speed components of the center of gravity (CoG) of the vehicle; and the 3 angular speeds associated with the local axes. All of them can be easily measured using commercially available sensors such as Inertial Measurement Units (IMU), Global Positioning Systems (GPS), and steering angle sensors. The output data is composed of the vertical force of the selected tire. The structure of the ANFIS designed for each wheel is detailed in Figure 5.

The proposed ANFIS estimator is composed of 4 layers. In the first of them 7 membership functions for each input have been developed. These membership functions are of Gaussian type, as they provide better precision than triangular ones [32, 35]. In the second one, the rules established by the learning process appear. In the third layer the ratio calculation and 


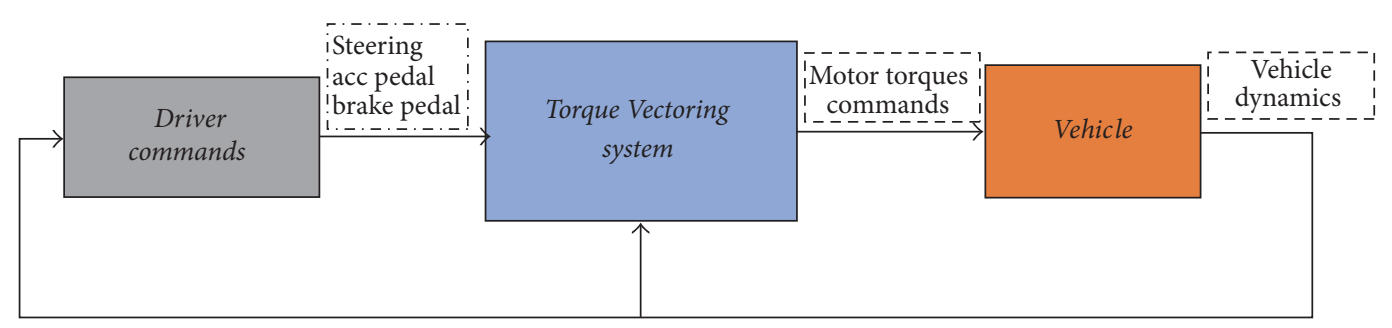

Figure 6: General test framework, based on Dynacar [36, 37].

TABle 5: Parameters of the hybrid method training.

\begin{tabular}{lc}
\hline Range of influence & 0.5 \\
Squash factor & 1.25 \\
Accept ratio & 0.5 \\
Reject ratio & 0.15 \\
\hline
\end{tabular}

normalisation are carried out to finally, in the fourth layer, add all the signals.

The method selected for the generation of the fuzzy inference system is Subclustering, due to the high number of inputs. The training method chosen is the hybrid method, which is a combination of least squares and backpropagation gradient descent method. The parameters of this process are detailed in Table 5.

The data used for the training and testing of the proposed ANFIS structure have been obtained from a simulation of a vehicle running on the Nurburgring circuit during one lap (simulation time of $800 \mathrm{~s}$ and sample time of $50 \mathrm{~ms}$ ). The simulation has been obtained from the high fidelity vehicle dynamics simulator Dynacar [36], where, for each vertical tire force estimation, the aforementioned 10 variables have been captured.

2.4. Longitudinal Torque Distribution Algorithm. The proposed longitudinal torque distribution approach calculates the longitudinal torque distribution percentage $\tau_{\text {long }}$ to be applied to the wheels of each axle (front and rear) considering the vertical normal forces generated by the wheels at each axle $f_{\text {wheels }}$. This way, if $\tau_{\text {long }}=0$, all the torque will be provided by the wheels of the rear axle, and if $\tau_{\text {long }}=1$, all the torque will be provided by the wheels of the front axle.

This way, this subsystem allows sending greater torque commands to the motors whose wheels have more grip. For that purpose, a simple but effective torque distribution algorithm is proposed, based on the maximum normal force that can be applied in an axle (the front one has been taken as reference). This way,

$$
\tau_{\text {long }}=\frac{f_{\text {wheels }}}{f_{\max }},
$$

where $f_{\text {wheels }}$ is the combination of the vertical forces of each wheel of an axle (left and right), given by the vertical tire forces estimator, and $f_{\max }$ is the maximum normal force that can be applied in the front axle (this is, considering that the whole mass is actuating only in one axle). For the study case selected, this final value is $20208 \mathrm{~N}$.

2.5. Motor Torque Calculation. This subsystem calculates the exact motor torque command to be applied to each wheel $\tau_{i j}$, based on the torque requested by the driver using the throttle $\tau$, and the longitudinal $\tau_{\text {long }}$ and lateral $\tau_{\text {lat }}$ torque distribution percentages:

$$
\begin{aligned}
\tau_{\text {fl }} & =\tau \tau_{\text {long }} \tau_{\text {lat }} \\
\tau_{\text {fr }} & =\tau \tau_{\text {long }}\left(1-\tau_{\text {lat }}\right) \\
\tau_{\text {rl }} & =\tau\left(1-\tau_{\text {long }}\right) \tau_{\text {lat }} \\
\tau_{\text {rr }} & =\tau\left(1-\tau_{\text {long }}\right)\left(1-\tau_{\text {lat }}\right) .
\end{aligned}
$$

\section{Validation Framework}

In this section the validation methodology used is explained, including the selected vehicle, the simulation environment, and the proposed manoeuvres and testing scenarios.

Figure 6 shows a general overview of the implemented control concept. Three main blocks can be considered: first, the driver command, which is generated by an automated driving algorithm that simulates the behaviour of a standard driver, guaranteeing that the performance of each test is not dependent on the driver; second, the control block, which includes the intelligent Torque Vectoring approach detailed in the previous section, with all subsystems; finally, the vehicle model, which simulates the highly nonlinear dynamics of the vehicle using Dynacar software [36] and provides the time evolution of the measurable variables.

3.1. Dynacar High Fidelity Dynamics Simulator. The vehicle model is implemented in Dynacar, which is a high fidelity vehicle dynamics simulation platform developed by Tecnalia Research \& Innovation [36]. This vehicle simulation software has been validated through several racetrack tests $[37,38]$ and it can be used in a model-in-the-loop framework to test the performance of different automotive aimed control systems.

One of the features of Dynacar is the possibility of activating an automated driver mode, which simulates a standard driver. This allows reducing the effect of the driver ability when analyzing the results of the developed ADAS and, hence, allowing better comparison.

Dynacar's vehicle physical model simulation engine is based on a multibody model and integrated in C code [39]. 
TABLE 6: Vehicle principal characteristics.

\begin{tabular}{lc}
\hline Mass $[\mathrm{kg}]$ & 1830 \\
$I x\left[\mathrm{kgm}^{2}\right]$ & 928.1 \\
$I y\left[\mathrm{kgm}^{2}\right]$ & 2788.5 \\
$I z\left[\mathrm{kgm}^{2}\right]$ & 3234.0 \\
Wheelbase $[\mathrm{m}]$ & 3.05 \\
Front axis track $[\mathrm{m}]$ & 1.6 \\
Rear axis track $[\mathrm{m}]$ & 1.6 \\
\hline
\end{tabular}

This $\mathrm{C}$ code has also been implemented in Simulink. The solver is run with a sample time of $1 \mathrm{~ms}$. Tires are modelled using implementation of the Pacejka "Magic Formula" model, extensively used by most car manufacturers as an industry standard for vehicle model simulations [18].

Table 6 shows the main characteristics of the vehicle, which is an E-Class vehicle.

3.2. Controller Implementation. The control block is implemented in a Xilinx Zynq XC7Z020 SoC, whose inputs and outputs are connected to Dynacar. This allows testing the real-time performance of the proposed intelligent TV control approach and does not require the use of a whole vehicle thanks to the Dynacar's model-in-the-loop approach.

The selected hardware is composed of two parts. The first is the programmable logic part, which is a full FPGA. And the other part is the processing system, which is composed of an ARM CPU of two cores and $800 \mathrm{MHz}$ clock rate. In addition, this board has several I/O peripherals, such as digital and analog inputs/outputs ports and communication buses.

The ARM core has been used to implement the different subsystems of the proposed intelligent TV approach (Figure 2) proposed in the previous section. For that purpose, the developed approach has been implemented first in Matlab Simulink and then compiled to $\mathrm{C}$ code that runs in the ARM processor.

3.3. Manoeuvres and Scenarios. Dynacar's framework allows simulating and testing the developed intelligent TV controller in different scenarios and with a set of different standardized manoeuvres: a skid-pad [40] (Figure 7) and a double lane change manoeuvre [41] (Figure 8).

On the one hand, the objective of the skid-pad test is to measure the car's cornering ability on a flat surface while making a constant-radius turn. This test is one of the FSAE Dynamics Events [40], but, as it is designed for formula type vehicles, it is necessary to adapt it to a passenger vehicle. In that sense, the diameters of the circles have been modified considering the rules for road designs [42]. These rules state that for a speed limit of $40 \mathrm{~km} / \mathrm{h}$ the minimum radius of a curve has to be 60 meters. However, as this radius is defined to ensure the stability of the car at that speed, in this work the radius has been reduced in order to take the vehicle to its limits and then be able to evaluate the correct performance of the developed controller. Therefore, it has been decreased until 20 meters, converting the test into a challenging scenario for an E-Class vehicle.
On the other hand, the double lane change manoeuvre is detailed in the ISO 3888 specification [41] (Figure 8). In this test, the vehicle enters the course at a particular speed and the throttle is released. The driver then attempts to negotiate the course without striking the cones. The test speed is progressively increased until either instability occurs or the course can no longer be negotiated successfully. Such a severe manoeuvre effectively demonstrates the cornering capability of a vehicle when driving at the friction limit in both directions and, therefore, many car manufacturers and research institutions consider this test to be a suitable manoeuvre for assessing advanced vehicle dynamics control systems. This manoeuvre is typically performed as a closedloop driving test and is used to adjust the dynamics of a vehicle based on the subjective evaluations of professional drivers.

\section{Results}

In this section the results obtained during the validation of the developed intelligent TV control approach are analyzed. For that purpose, first the proposed ANFIS vertical force estimator is validated with the results obtained with Dynacar and the analytical estimator proposed in [43], in order to demonstrate its accuracy. Then, the overall intelligent TV approach including all subsystems (Figure 2) is validated, comparing its results with a more traditional solutions. In the case of the intelligent TV algorithm its performance is compared with a PID TV controller based on [6] with a constant longitudinal torque distribution, while the ANFIS estimator's performance is compared with the analytical estimator proposed in [43].

4.1. ANFIS-Based Vertical Tire Force Estimation. In order to test the effectiveness of the approach, the data obtained from the proposed estimator is compared with (a) Dynacar's internal high fidelity tire model and (b) the model-based analytical estimator proposed in [43], whose results have been validated in the cited work, but it requires data from variables that are difficult to measure. The aforementioned two manoeuvres have been used to validate the force estimator.

Figures 9 and 10 and Tables 7 and 8 show the results obtained for each manoeuvre and each wheel. They show that the ANFIS estimator is able to reduce the error between 38\% and $79 \%$ compared with the analytical approach (depending on the wheel and the manoeuvre), being able to eliminate the peaks obtained by the analytical estimator due to transient conditions. It has to be noted that the errors obtained for the analytical model correspond to the errors obtained in [43].

In addition, the real-time performance of the proposed estimator has been analyzed, requiring $0.9 \mathrm{~ms}$ to run, which is appropriate for automotive applications.

4.2. Intelligent Torque Vectoring System. In order to validate the ability of the proposed intelligent TV approach to enhance the dynamic handling, first, the results associated with the skid-pad test will be analyzed.

In order to determine the effectiveness of the approach, the critical speed of the vehicle has to be defined first. This 
FSAE skid-pad layout

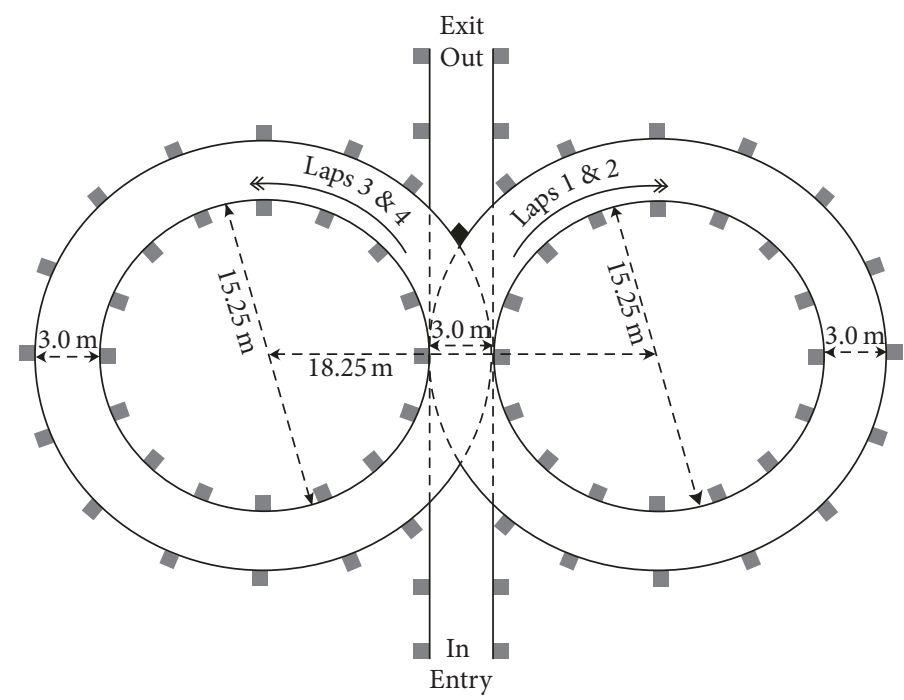

Placement of pylons/cones

- Pylons/cones to be removed for exit

Figure 7: Skid-pad test [40].

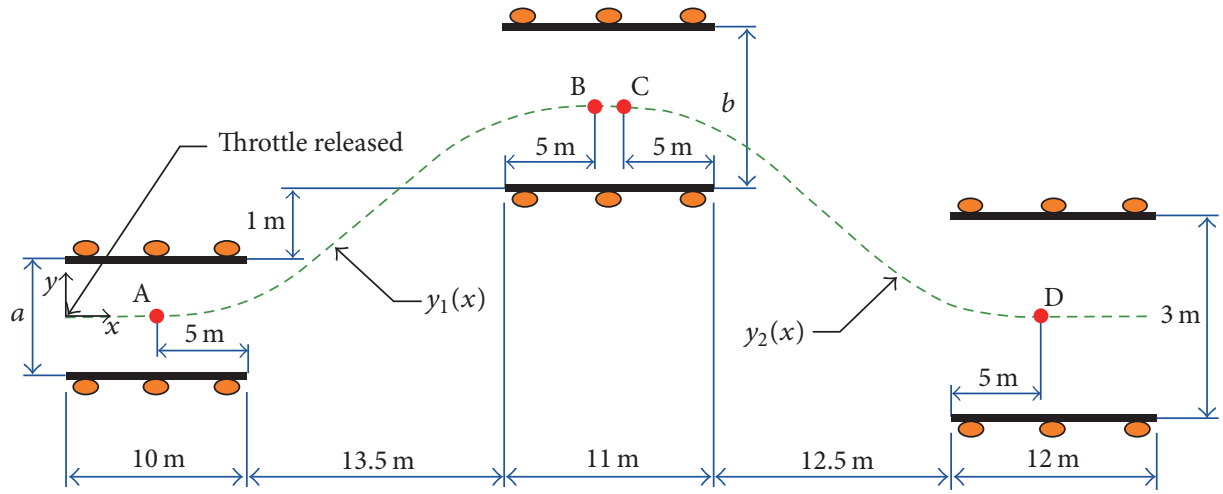

$a=1.1 \times$ vehicle width $+0.25 \mathrm{~m}$

$b=$ vehicle width $+1.0 \mathrm{~m}$

Figure 8: Double lane change test [41].

TABLE 7: Skid-pad results.

\begin{tabular}{cccc}
\hline & & ANFIS & Model \\
\hline \multirow{2}{*}{ FL } & RMSE & 250.192 & 702.36 \\
& NRMSE & 2.37 & 6.656 \\
FR & RMSE & 211.58 & 698.56 \\
& NRMSE & 2.015 & 6.635 \\
RL & RMSE & 143.544 & 412.77 \\
& NRMSE & 2.0474 & 5.8874 \\
RR & RMSE & 245.871 & 434.997 \\
& NRMSE & 3.4764 & 5.5628 \\
\hline
\end{tabular}

TABLE 8: Double lane change results.

\begin{tabular}{cccc}
\hline & & ANFIS & Model \\
\hline FL & RMSE & 114.23 & 787.905 \\
& NRMSE & 1.3491 & 5.944 \\
FR & RMSE & 151.0323 & 775.32 \\
& NRMSE & 1.607 & 5.185 \\
RL & RMSE & 107.986 & 412.77 \\
& NRMSE & 1.4629 & 6.782 \\
RR & RMSE & 164.2598 & 434.997 \\
& NRMSE & 2.0457 & 5.982 \\
\hline
\end{tabular}



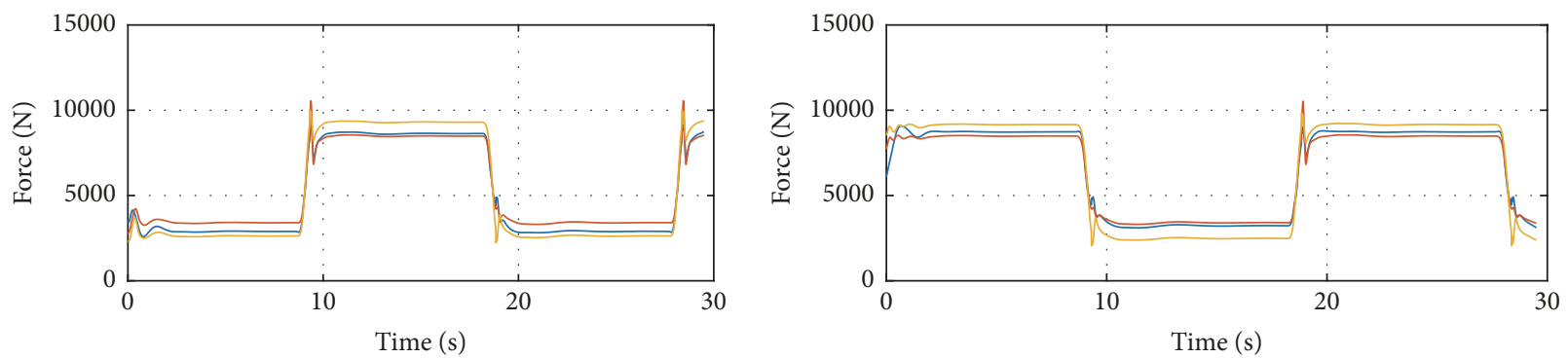

- ANFIS (FL)

— Dynacar (FL)

_ Analytical model (FL)

- ANFIS (FR)

— Dynacar (FR)

__ Analytical model (FR)
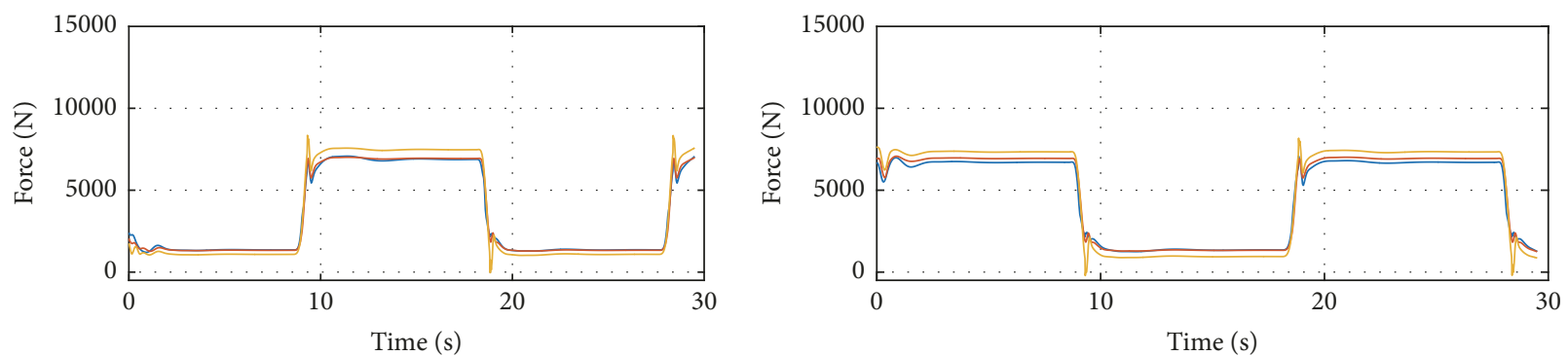

— ANFIS (RL)

- ANFIS (RR)

— Dynacar (RL)

Analytical model (RL)

— Dynacar (RR)

Analytical model (RR)

FIgure 9: $F z$ estimation, skid-pad.
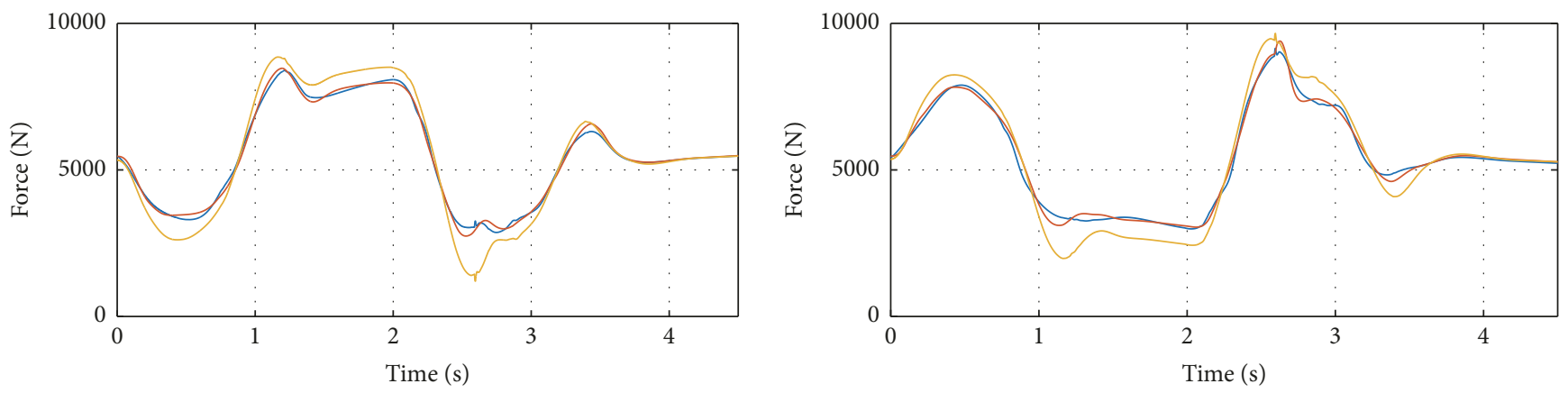

- ANFIS (FL)

- Dynacar (FL)

_ Analytical model (FL)

- ANFIS (FR)

— Dynacar (FR)

- Analytical model (FR)
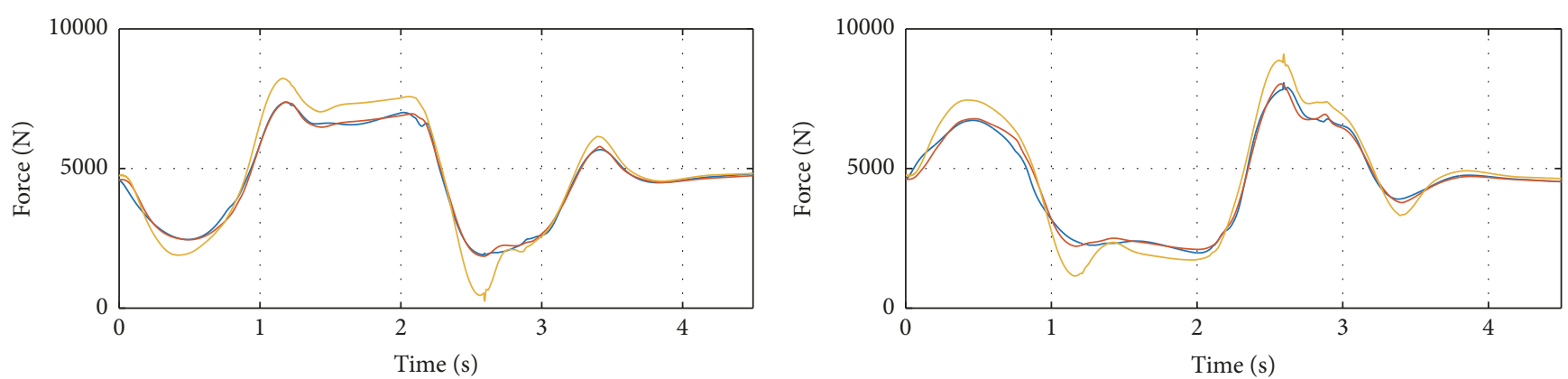

- ANFIS (RL)

Dynacar (RL)

ANFIS (RR)

Dynacar (RR)

_ Analytical model (RL)

_ Analytical model (RR)

Figure 10: $F z$ estimation, double lane change. 


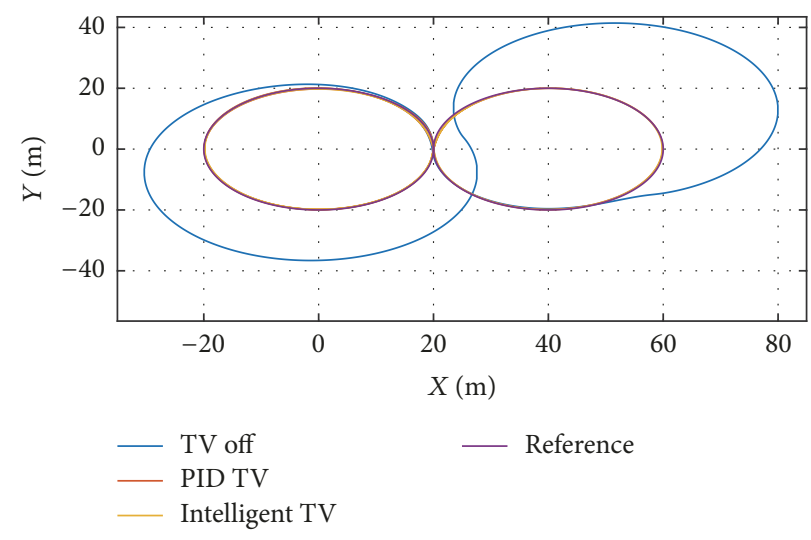

FIGURE 11: Skid-pad trajectory.
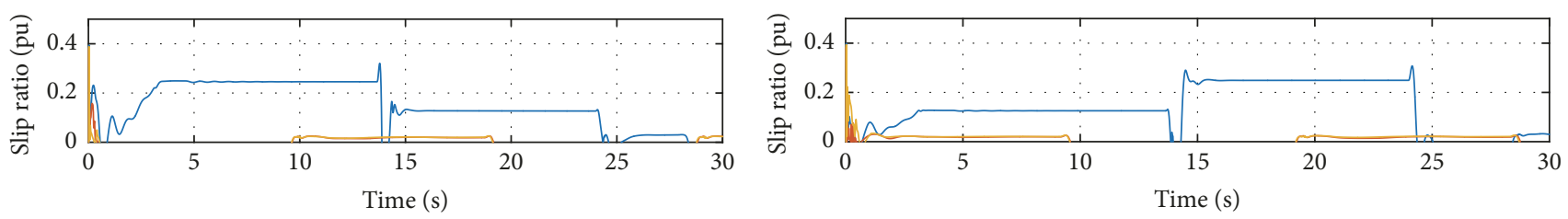

— TV off (FL)

— PID TV (FL)

_ Intelligent TV (FL)

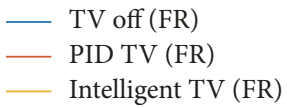

Figure 12: Wheels slip ratio.
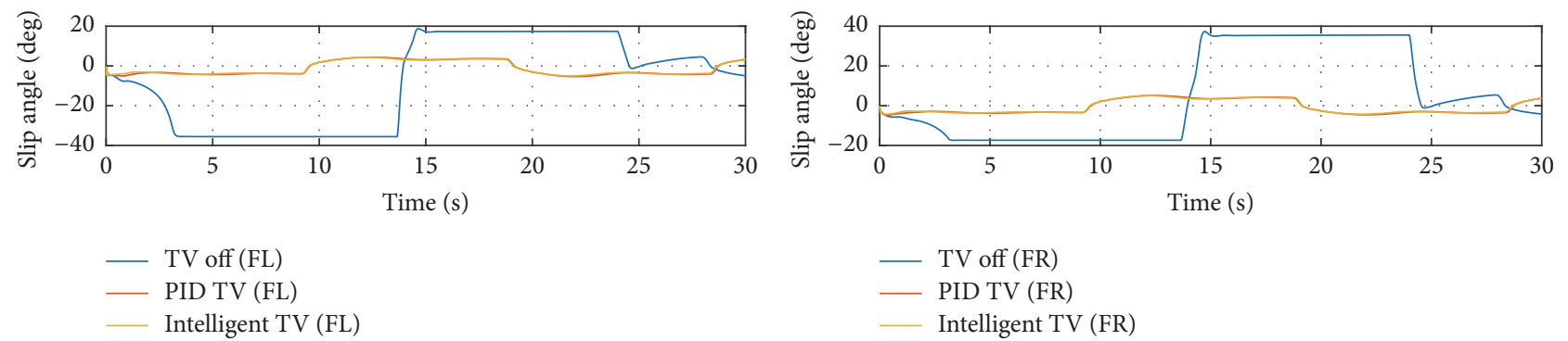

FIGURE 13: Wheels slip angles.

critical speed is the maximum speed that allows the vehicle to perform the skid-pad test correctly with no TV control. For that purpose, no TV system has been activated, and the skidpad test has been carried out increasing the speed in each test until the vehicle has not been able to follow the reference trajectory. This critical speed has been experimentally defined as $47 \mathrm{~km} / \mathrm{h}$, providing a theoretical lateral acceleration of $0.86 \mathrm{~g}$.

Once this critical speed limit is detected, the skid-pad test has been executed activating the proposed intelligent TV approach and a PID based TV approach. Results are shown in Figure 11. It can be appreciated that when no TV controller is active, the vehicle is not able to track the desired trajectory, due to understeering, but that TV approaches allow executing this test even at the critical speed.

The undesirable behaviour at the critical speed when no TV is activated can be further appreciated in Figures 12 and
13, which show the slip ratio and slip angle of the front wheels. As it can be seen, these wheels cannot transmit the requested force to the road and they slip, which can be detected by high values of the slip ratio and angles in the TV off case. Furthermore, when either TV control is active (fuzzy TV and PID TV), slip ratio and slip angle are reduced to $19 \%$ and $23^{\circ}$, respectively, which implies higher traction forces on the tires. This implies higher speeds in the skid-pad tests, as shown in Figure 14. Moreover, higher lateral acceleration and yaw rate values are achieved without losing stability as illustrated in Figures 15 and 16.

Figure 16 illustrates the yaw rate evolution of the vehicle. As it can be seen, the proposed intelligent Torque Vectoring approach provides a correct tracking of the reference, while reducing the overshoots. This allows increasing the cornering ability of the vehicle, reducing the risk of underand oversteering. In fact, the difference between the yaw rate 


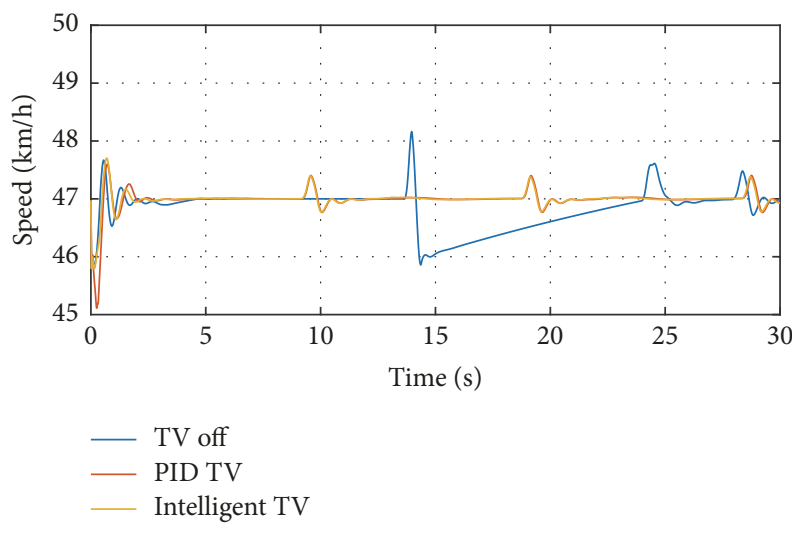

Figure 14: Vehicle speed.

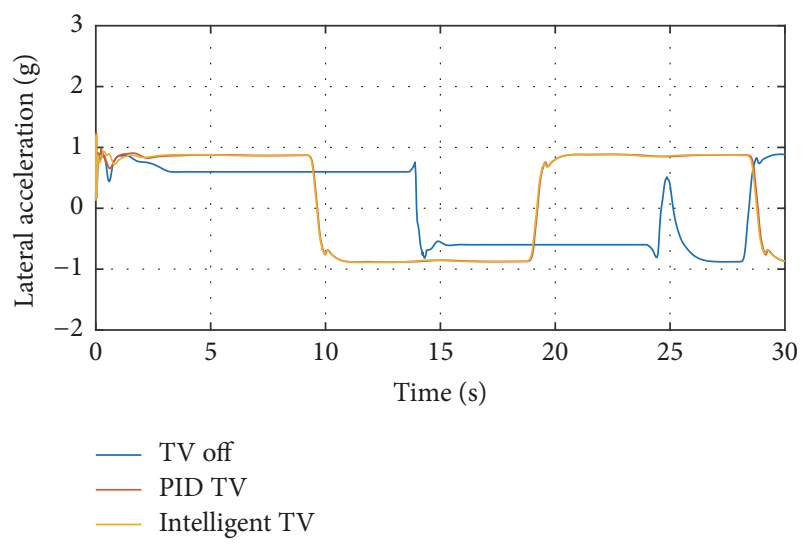

FIGURE 15: Vehicle lateral acceleration.

TABLE 9: Mechanical energy comparison.

\begin{tabular}{lc}
\hline & Energy mech $[\mathrm{kWh}]$ \\
\hline No TV & 0.4219 \\
PID TV & 0.2091 \\
Intelligent TV & 0.1895 \\
\hline
\end{tabular}

reference and the obtained values for the case where the TV is deactivated shows understeering behaviour as has been explained.

Moreover, if a mechanical energy consumption analysis is carried out, the proposed intelligent Torque Vectoring approach allows not only a correct yaw rate tracking, increased cornering ability, and reduced slip ratio, but also an increase in the efficiency of the vehicle. Efficiency results are shown in Table 9, where the proposed approach is compared with the PID based TV and no TV cases. As can be seen, an increase of $10 \%$ can be achieved.

After analyzing the skid-pad performance, the double lane change scenario will be studied. In order to perform this test, an initial speed of $50 \mathrm{~km} / \mathrm{h}$ has been selected, and a constant torque reference has been applied to the motors ( $2300 \mathrm{Nm}$ total torque). This provides a longitudinal acceleration of $0.35 \mathrm{~g}$ approximately, allowing to obtain a final

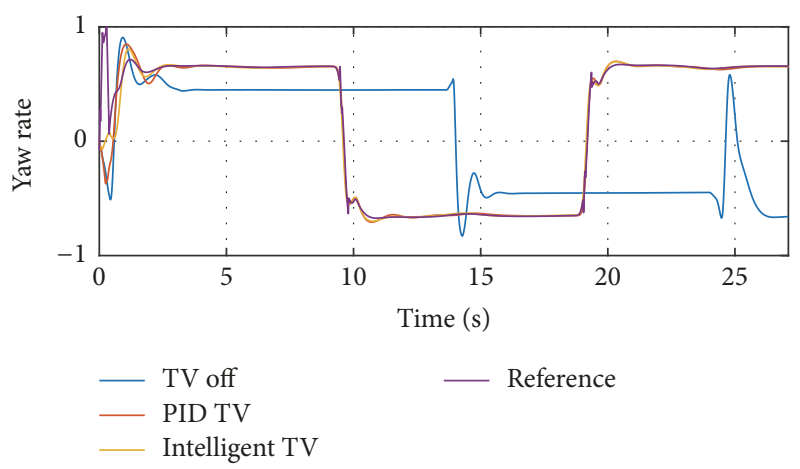

FIGURE 16: Yaw rate tracking.

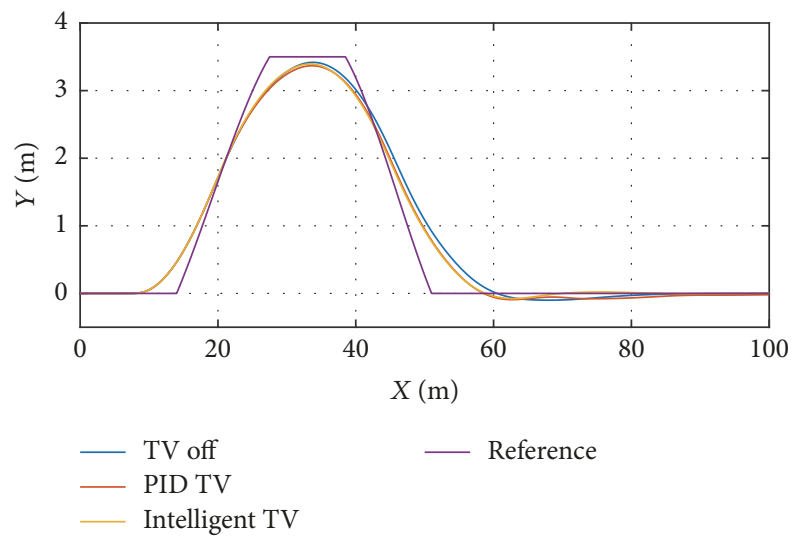

FIGURE 17: Double lane change trajectory.

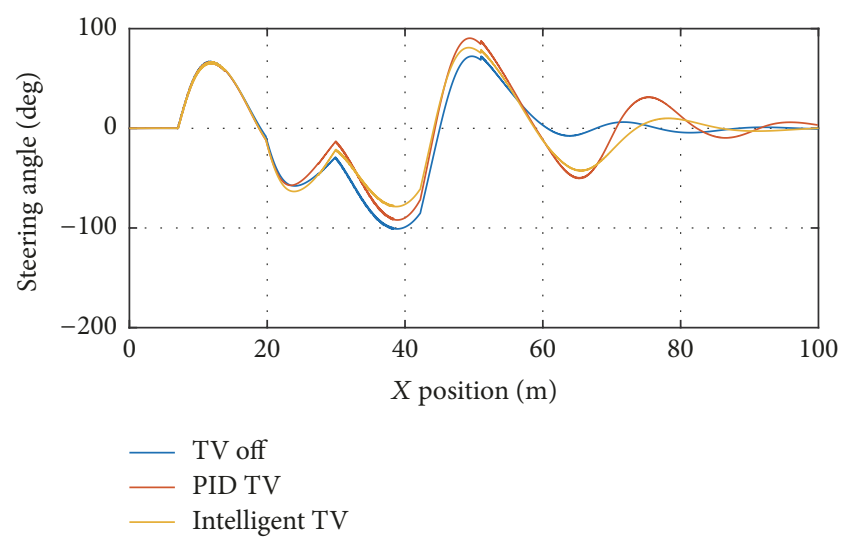

FIGURE 18: Steering angle.

speed of almost $90 \mathrm{~km} / \mathrm{h}$, covering the most common speed range of passenger vehicles in medium speed roads.

Simulation results for this scenario are shown from Figures 17-20. Figure 17 shows the trajectory followed by the vehicle for each case. As can be seen in this figure, the trajectory when the proposed TV approach is activated is closer to the lateral double lane change manoeuvre reference, demonstrating a vehicle handling improvement in such a challenging manoeuvre. In addition, Figure 18 shows that this 

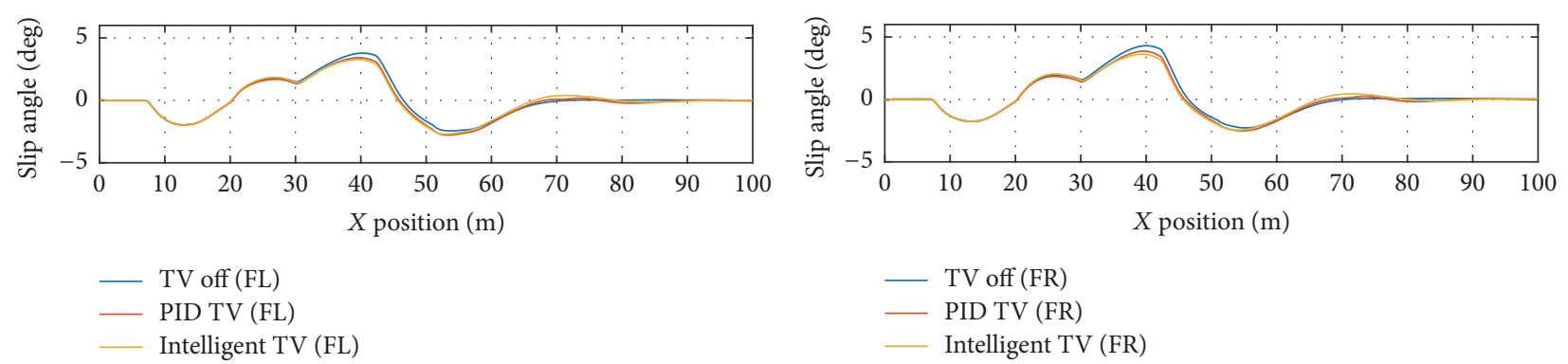

FIGURE 19: Wheels slip angles.
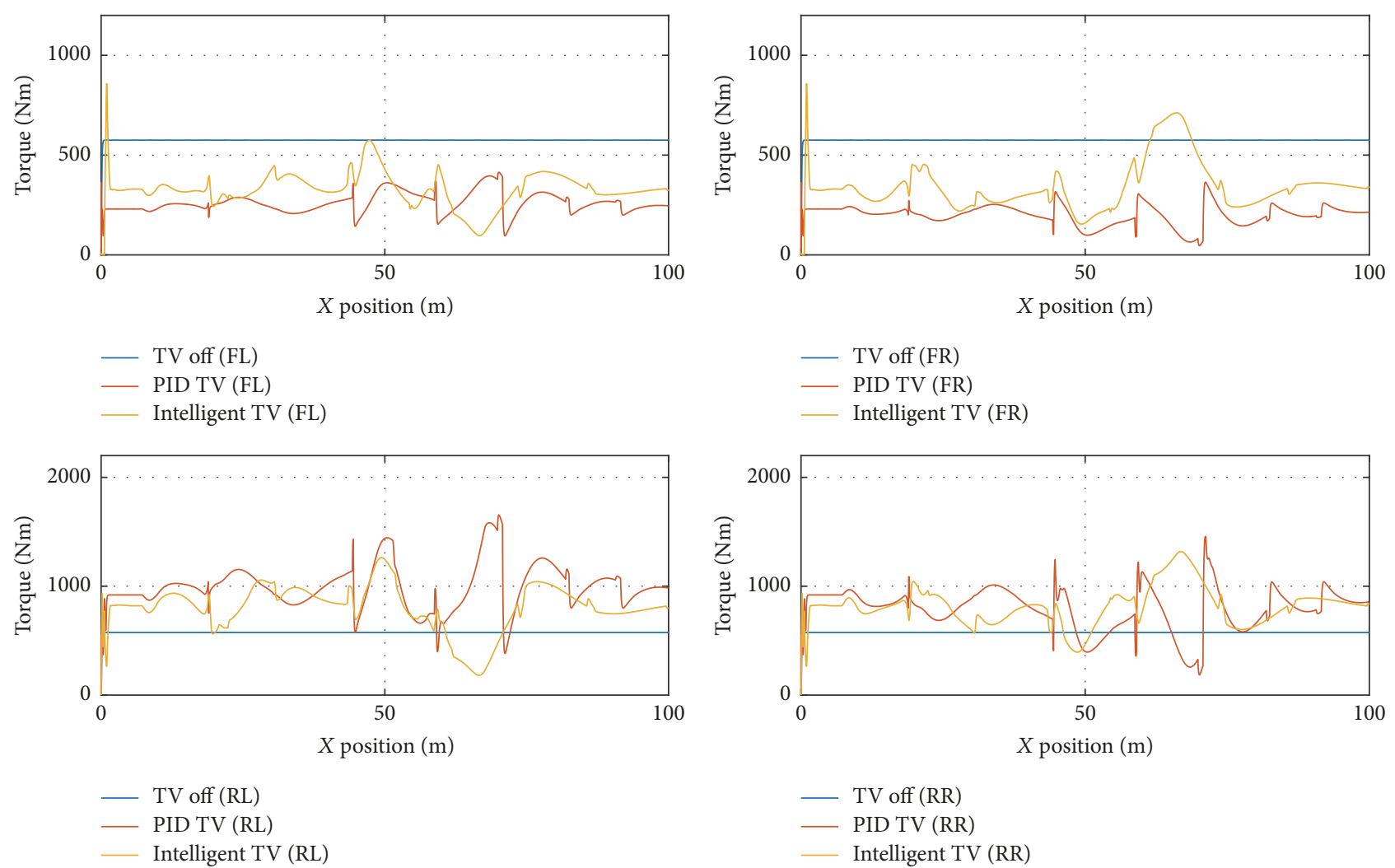

Figure 20: Wheel torque.

better tracking is achieved with lower values of steering angle, which means a reduction of the slip angle of about $1.5^{\circ}$ as can be seen in Figure 19. The maximum reduction of this value is achieved by the proposed intelligent TV controller, which allows maximizing the forces transmitted to the road. Finally, wheel torque is illustrated in Figure 20, showing the correct torque distribution made by the controller designed.

\section{Conclusions}

The development of real-time capable, accurate, and efficient ADAS is a key issue for the development of vehicles with independent in-wheel motors. In this work a novel intelligent Torque Vectoring (TV) system, composed of a neuro-fuzzy vertical tire forces estimator and a fuzzy yaw moment controller, has been proposed.
The proposed approach considers both lateral and longitudinal torque distributions. The longitudinal distribution is based on a neuro-fuzzy vertical tire forces estimator that is based exclusively on measurable variables, which is an important contribution compared with the existing estimators. The estimated forces are used to determine the percentage of torque to be applied to the wheels of the rear and front axles, so that the maximum grip can be achieved.

On the other hand, the lateral torque distribution is achieved using a fuzzy yaw moment controller. This controller allows distributing the torque laterally (right and left wheels), to minimize wheel slip and enhance cornering capabilities. The overall torque distribution is calculated by taking into account both distributions.

Results demonstrate the ability to enhance vehicle dynamics of the intelligent Torque Vectoring System in 
various scenarios. On the one hand, it was able to increase the stability in an evasive manoeuvre, such as double lane change, allowing the vehicle to follow better the desired trajectory, which is a critical safety issue in such manoeuvre. On the other hand, in the skid-pad test, a significant wheel slip ratio and slip angle reduction (19\% and $23^{\circ}$, resp.) have been shown, resulting in an understeering behaviour reduction. This has allowed the vehicle to better match the yaw rate reference (33\% error reduction) and then be able to follow the desired trajectory, demonstrating the cornering improvement provided by the correct torque distribution. Additionally, the proposed intelligent TV algorithm presents an improvement regarding a more traditional approach of the state of the art, providing more efficient driving (10\% mechanical energy consumption reduction).

Future work will include a more sophisticated design for the use of the estimated tire vertical forces in the intelligent Torque Vectoring controller, resulting in a more elaborate controller, to improve its performance. Moreover, the implementation of the TV System in the logical part of a SoC will be considered in order to decrease its cycle time.

\section{Conflicts of Interest}

The authors declare that there are no conflicts of interest regarding the publication of this paper.

\section{Acknowledgments}

The research leading to these results has been supported by the ECSEL Joint Undertaking under Grant agreement no. 662192 (3Ccar). This Joint Undertaking receives support from the European Union Horizon 2020 research and innovation program and the ECSEL member states.

\section{References}

[1] ACEA, European Automobile Manufacturers Association New passenger car registrations by alternative fuel type in the European Union Q4 2015, 2016.

[2] A. Al-Hmouz, J. Shen, R. Al-Hmouz, and J. Yan, "Modeling and simulation of an Adaptive Neuro-Fuzzy Inference System (ANFIS) for mobile learning," IEEE Transactions on Learning Technologies, vol. 5, no. 3, pp. 226-237, 2012.

[3] M. Nikowitz, Advanced Hybrid and Electric Vehicles, Springer, 2016.

[4] A. Broggi, A. Zelinsky, Ü. Özgüner, and C. Laugier, "Intelligent Vehicles," in Springer Handbook of Robotics, B. Siciliano and O. Khatib, Eds., Springer, Cham, Switzerland, 2016.

[5] S. Inoue, T. Ozawa, H. Inoue, P. Raksincharoensak, and M. Nagai, "Cooperative lateral control between driver and ADAS by haptic shared control using steering torque assistance combined with direct yaw moment control," in Proceedings of the 19th IEEE International Conference on Intelligent Transportation Systems (ITSC '16), pp. 316-321, Brazil, November 2016.

[6] L. De Novellis, A. Sorniotti, P. Gruber, and A. Pennycott, "Comparison of feedback control techniques for torque-vectoring control of fully electric vehicles," IEEE Transactions on Vehicular Technology, vol. 63, no. 8, pp. 3612-3623, 2014.
[7] K. Shimada and Y. Shibahata, "Comparison of three active chassis control methods for stabilizing yaw moments," $S A E$ Technical Papers, 1994.

[8] L. De Novellis, A. Sorniotti, P. Gruber, L. Shead, V. Ivanov, and K. Hoepping, "Torque vectoring for electric vehicles with individually controlled motors: State-of-the-art and future developments," World Electric Vehicle Journal, vol. 5, no. 2, pp. 617-628, 2012.

[9] A. Haddoun et al., "Sliding mode control of EV electric differential system," in Proceedings of the ICEM, Chania, Greece.

[10] A. Haddoun, M. E. H. Benbouzid, D. Diallo, R. Abdessemed, J. Ghouili, and K. Srairi, "Modeling, analysis, and neural network control of an EV electrical differential," IEEE Transactions on Industrial Electronics, vol. 55, no. 6, pp. 2286-2294, 2008.

[11] K. Jalali, T. Uchida, J. Mcphee, and S. Lambert, "Integrated stability control system for electric vehicles with in-wheel motors using soft computing techniques," SAE International Journal of Passenger Cars - Electronic and Electrical Systems, vol. 2, no. 1, pp. 109-119, 2009.

[12] H. Kahveci, H. I. Okumus, and M. Ekici, "An electronic differential system using fuzzy logic speed controlled in-wheel brushless DC motors," in Proceedings of the 4th International Conference on Power Engineering, Energy and Electrical Drives, pp. 881-885, Turkey, May 2013.

[13] D. E. Williams and W. M. Haddad, "Nonlinear control of roll moment distribution to influence vehicle yaw characteristics," IEEE Transactions on Control Systems Technology, vol. 3, no. 1, pp. 110-116, Mar 1995.

[14] Y. Hu, L. Yang, B. Yan, T. Yan, and P. Ma, "An online rolling optimal control strategy for commuter hybrid electric vehicles based on driving condition learning and prediction," IEEE Transactions on Vehicular Technology, vol. 65, no. 6, pp. 43124327, 2016.

[15] I. Yang, S. Byun, B. Seo, D. Lee, and D. S. Han, "Integrated control systems of active front steering and direct yaw moment control using dynamic inversion," in Proceedings of the 2013 IEEE Intelligent Vehicles Symposium (IEEE IV '13), pp. 13031306, Australia, June 2013.

[16] C. Shen, G. Yin, and X. Jin, "Study on the active steering system with direct yaw-moment control for electric vehicle with electric motored wheels," in Proceedings of the 35th Chinese Control Conference (CCC '16), pp. 8945-8950, China, July 2016.

[17] E. Siampis, E. Velenis, and S. Longo, "Model Predictive torque vectoring control for electric vehicles near the limits of handling," in Proceedings of the European Control Conference (ECC '15), pp. 2553-2558, Austria, 2015.

[18] H. B. Pacejka, "Tyre and Vehicle Dynamics, BH, 2005”.

[19] J. Kim, H. Lee, and S. Choi, "A robust road bank angle estimation based on a proportional-integral Hfilter," Proceedings of the Institution of Mechanical Engineers, Part D: Journal of Automobile Engineering, vol. 226, no. 6, pp. 779-794, 2012.

[20] R. Ghandour, A. Victorino, M. Doumiati, and A. Charara, "Tire/road friction coefficient estimation applied to road safety," in Proceedings of the 18th Mediterranean Conference on Control and Automation (MED '10), pp. 1485-1490, June 2010.

[21] W. Cho, J. Yoon, S. Yim, B. Koo, and K. Yi, "Estimation of tire forces for application to vehicle stability control," IEEE Transactions on Vehicular Technology, vol. 59, no. 2, pp. 638649, 2010.

[22] M. Doumiati, A. Victorino, D. Lechner, G. Baffet, and A. Charara, "Observers for vehicle tyre/road forces estimation: 
Experimental validation," Vehicle System Dynamics, vol. 48, no. 11, pp. 1345-1378, 2010.

[23] S. Antonov, A. Fehn, and A. Kugi, "Unscented Kalman filter for vehicle state estimation," Vehicle System Dynamics, vol. 49, no. 9, pp. 1497-1520, 2011.

[24] M. Doumiati, A. Victorino, A. Charara, and D. Lechner, "Lateral load transfer and normal forces estimation for vehicle safety: Experimental test," Vehicle System Dynamics, vol. 47, no. 12, pp. 1511-1533, 2009.

[25] A. Rabhi, N. K. M'Sirdi, and A. Elhajjaji, "Estimation of contact forces and tire road friction," in Proceedings of the Mediterranean Conference on Control and Automation, pp. 1-6, 2007.

[26] M. Acosta and S. Kanarachos, "Tire lateral force estimation and grip potential identification using Neural Networks, Extended Kalman Filter, and Recursive Least Squares," Neural Computing and Applications, pp. 1-12, 2017.

[27] R. Jayachandran, S. D. Ashok, and S. Narayanan, "Fuzzy logic based modelling and simulation approach for the estimation of tire forces," in Proceedings of the 2013 International Conference on Design and Manufacturing, vol. 64, pp. 1109-1118, India, July 2013.

[28] H. Wallentowitz, Vertical and Lateral Dynamics of Passenger Vehicles, Automotive Technology II, Institute of Automotive Engineering, Aachen University of Technology, Germany, 2005.

[29] L. A. Zadeh, "Fuzzy sets," Information and Control, vol. 8, no. 3, pp. 338-353, 1965.

[30] M. Blej and M. Azizi, "Comparison of Mamdani-type and Sugeno-type fuzzy inference systems for fuzzy real time scheduling," International Journal of Applied Engineering Research, vol. 11, no. 22, pp. 11071-11075, 2016.

[31] D. L. Milliken and W. F. Milliken, "Race Car Vehicle Dynamics," Society of Automotive Engineers, 1995.

[32] Y. Bai and D. Wang, "Fundamentals of Fuzzy Logic Control - Fuzzy Sets, Fuzzy Rules and Defuzzifications," in Advanced Fuzzy Logic Technologies in Industrial Applications, Advances in Industrial Control, Y. Bai, H. Zhuang, and D. Wang, Eds., Springer, London, UK, 2006.

[33] J. S. R. Jang, "ANFIS: adaptive-network-based fuzzy inference system," IEEE Transactions on Systems, Man, and Cybernetics, vol. 23, no. 3, pp. 665-685, 1993.

[34] M. Asadi, "Optimized Mamdani fuzzy models for predicting the strength of intact rocks and anisotropic rock masses," Journal of Rock Mechanics and Geotechnical Engineering, vol. 8, no. 2, pp. 218-224, 2016.

[35] K. M. Passino and S. Yurkovich, Fuzzy Control, 1997.

[36] Dynacar by Tecnalia, http://www.dynacar.es/en/home.php.

[37] A. Pena, I. Iglesias, J. J. Valera, and A. Martin, "Development and validation of Dynacar RT software, a new integrated solution for design of electric and hybrid vehicles," in Proceedings of the 26th Electric Vehicle Symposium (EVS 2012), pp. 2026-2032, USA, May 2012.

[38] M. Dendaluce, I. Iglesias, A. Martin, P. Prieto, and A. Peña, "Race-Track testing of a torque vectoring algorithm on a motorin-wheel car using a model-based methodology with a hil and multibody simulator setup," in Proceedings of the 19th IEEE International Conference on Intelligent Transportation Systems (ITSC 2016), pp. 2500-2505, Brazil, November 2016.
[39] J. Cuadrado, D. Vilela, I. Iglesias, A. Martín, and A. Peña, "A multibody model to assess the effect of automotive motor inwheel configuration on vehicle stability and comfort," in Proceedings of the ECCOMAS Thematic Conference on Multibody Dynamics, pp. 1083-1092, 2013.

[40] https://www.fsaeonline.com/.

[41] https://www.iso.org/standard/57253.html.

[42] Ministerio de Transportes y Comunicaciones, Manual De Carreteras Diseno Geometrico, 2014.

[43] A. Rezaeian, R. Zarringhalam, S. Fallah et al., "Novel tire force estimation strategy for real-time implementation on vehicle applications," IEEE Transactions on Vehicular Technology, vol. 64, no. 6, pp. 2231-2241, 2015. 


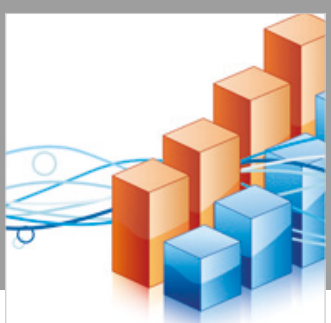

Advances in

Operations Research

\section{-n-m}
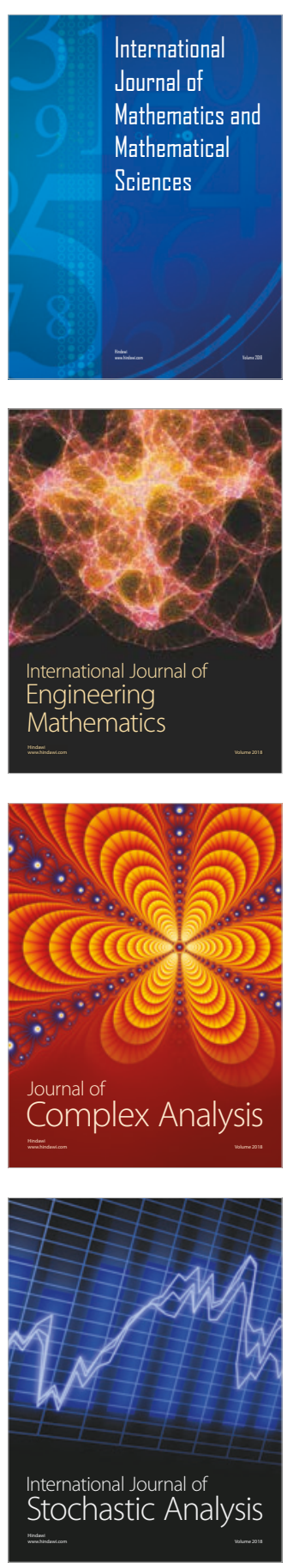
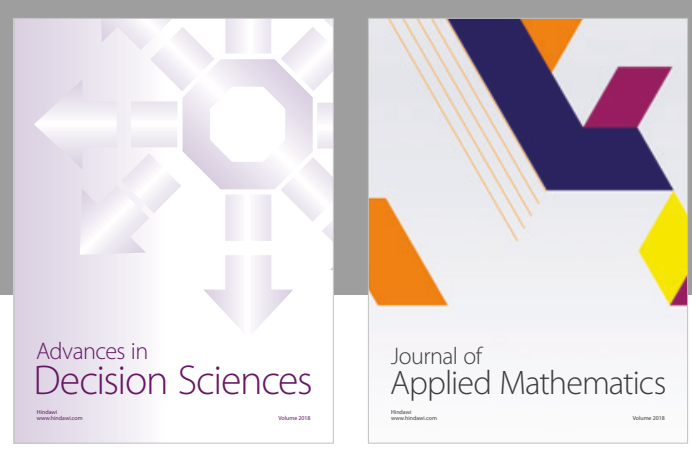

Journal of

Applied Mathematics
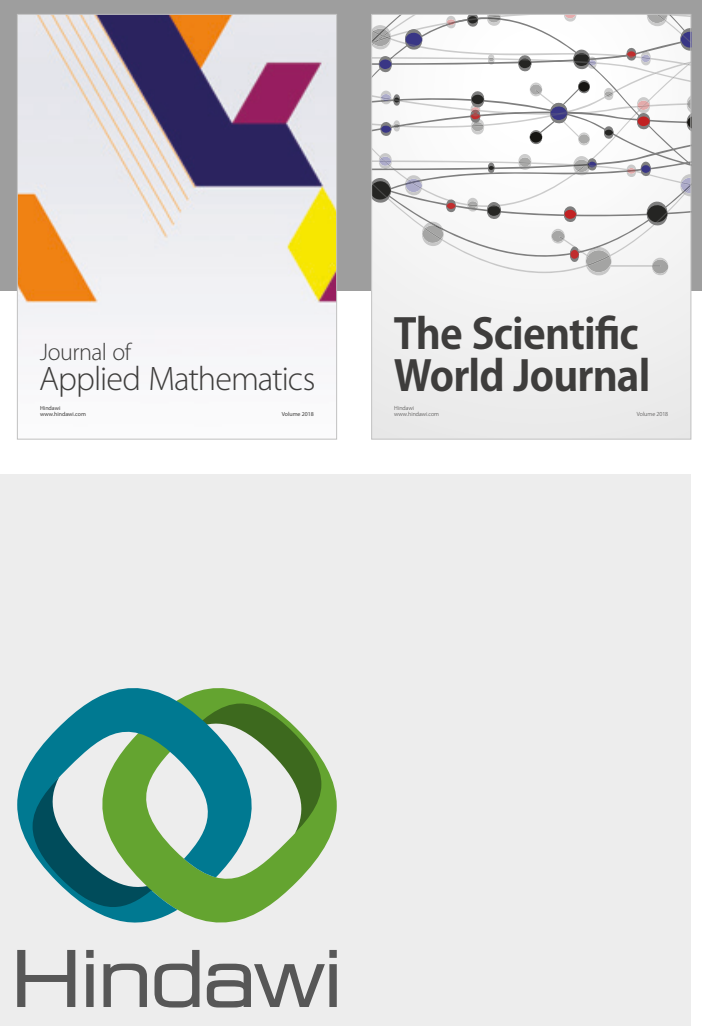

Submit your manuscripts at

www.hindawi.com

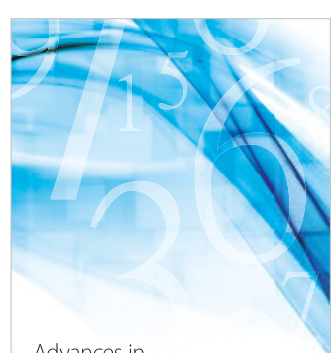

Advances in
Numerical Analysis
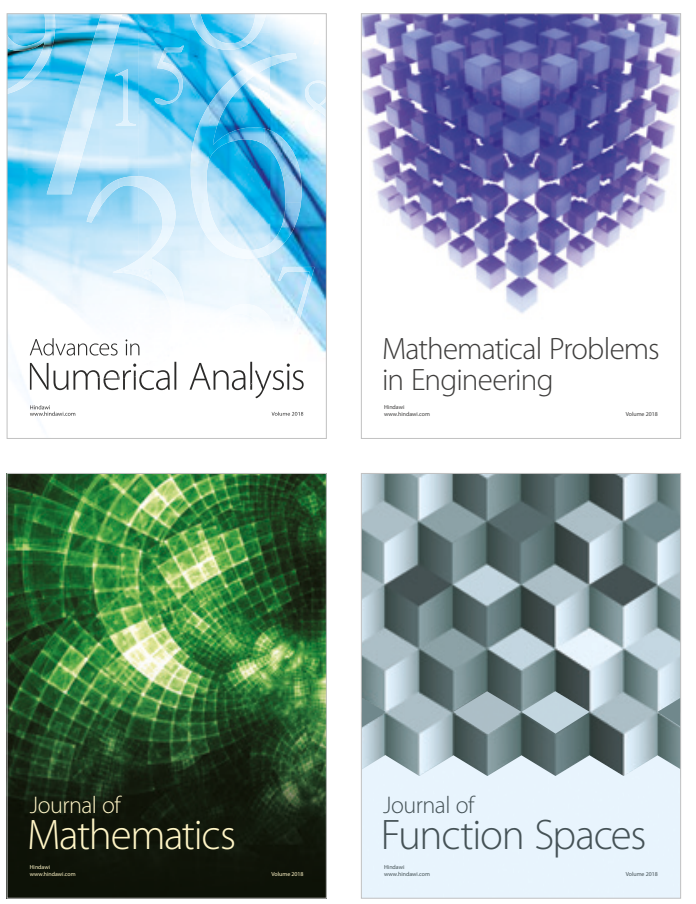

Mathematical Problems in Engineering

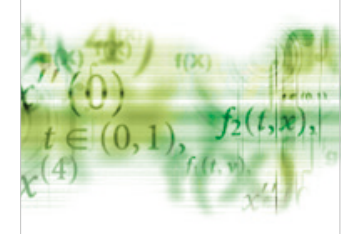

International Journal of

Differential Equations

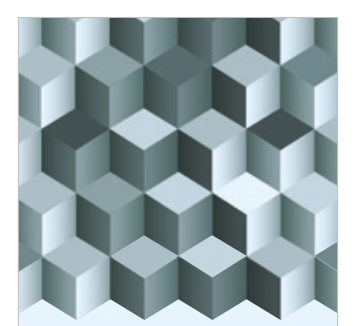

Journal of

Function Spaces
The Scientific

World Journal

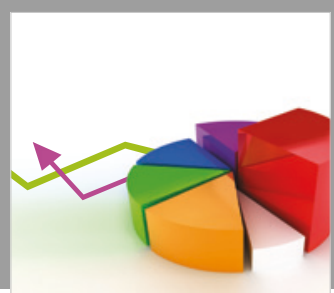

Journal of

Probability and Statistics
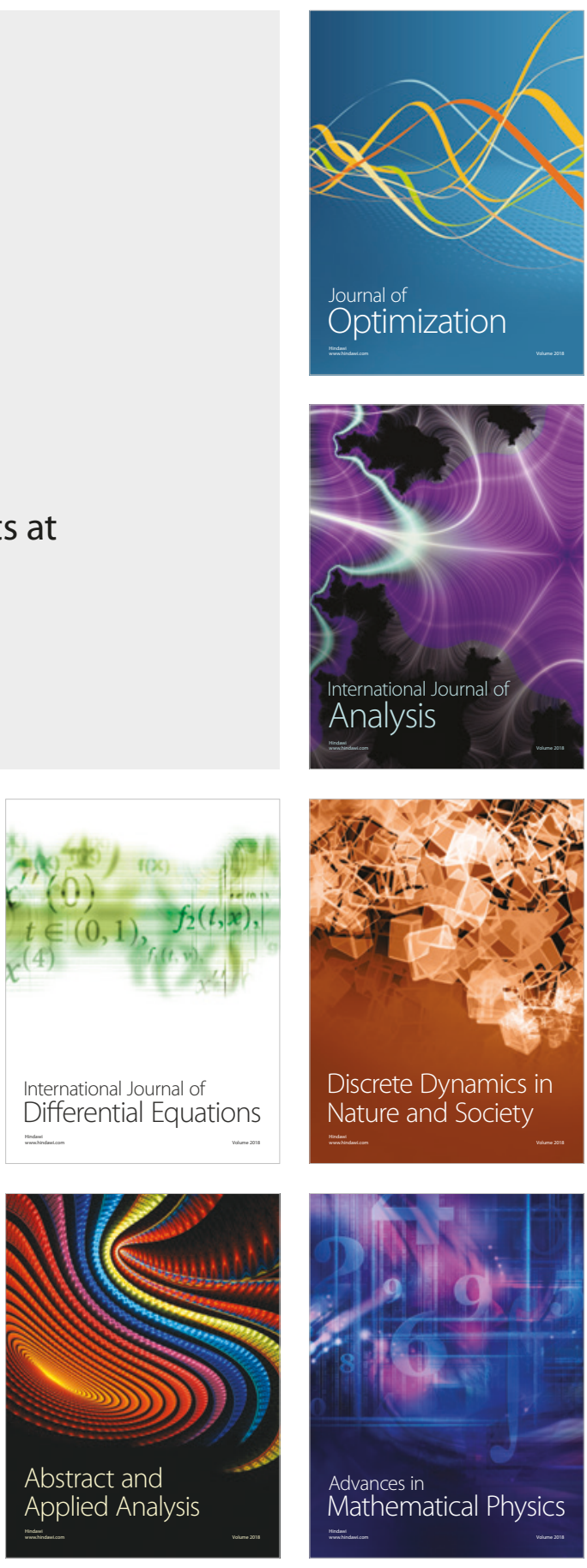